\title{
BMJ Open Factors associated with recruitment success in the phase 2 a study of aztreonam-avibactam development programme: a descriptive qualitative analysis among sites in Spain
}

Rosa M Jimenez-Rodriguez (1) ,' Guillermo Martín-Gutiérrez, ${ }^{2}$ Silvia Jiménez-Jorge, ${ }^{3}$ Clara M Rosso-Fernández, ${ }^{3}$ Luis Tallón-Aguilar, ${ }^{1}$ Cristina Roca-Oporto, ${ }^{4}$ Javier Padillo, ${ }^{1}$ Alison Luckey, ${ }^{5}$ Angela Cano, ${ }^{6}$ José López-Ruiz, ${ }^{7}$ Silvia Gómez-Zorrilla, ${ }^{8}$ Jaime Bonnín-Pascual, ${ }^{9}$ Lucía Boix-Palop, ${ }^{10}$ José Miguel Montejo, ${ }^{11}$ Julian Torre-Cisneros (D) , ${ }^{12}$ José Miguel Cisneros ${ }^{4}$

To cite: Jimenez-Rodriguez RM, Martín-Gutiérrez G, JiménezJorge S, et al. Factors associated with recruitment success in the phase 2a study of aztreonam-avibactam development programme: a descriptive qualitative analysis among sites in Spain. BMJ Open 2022;12:e051187. doi:10.1136/ bmjopen-2021-051187

- Prepublication history and additional supplemental material for this paper are available online. To view these files, please visit the journal online (http://dx.doi.org/10.1136/ bmjopen-2021-051187).

RMJ-R and GM-G contributed equally.

Received 02 April 2021 Accepted 13 January 2022

Check for updates

(C) Author(s) (or their employer(s)) 2022. Re-use permitted under CC BY-NC. No commercial re-use. See rights and permissions. Published by BMJ.

For numbered affiliations see end of article.

Correspondence to Dr Rosa M Jimenez-Rodriguez; ros_j_r@hotmail.com

\section{ABSTRACT}

Objective Successful clinical trials are subject to recruitment. Recently, the REJUVENATE trial, a prospective phase 2a open-label, single-arm interventional clinical trial conducted within the Innovative Medicines Initiativesupported Combatting Bacterial Resistance in EuropeCarbapenem Resistance project, was published, with $85 \%$ of the recruitment performed in Spain. We analysed the recruitment success in this trial by establishing a model of recruitment practice.

Methods A descriptive qualitative study was performed from May 2016 to October 2017 at 10 participating Spanish centres. Data were extracted from: (1) feasibility questionnaires to assess the centre's potential for patient enrolment; (2) delegation of responsibility records; (3) pre-screening records including an anonymised list of potentially eligible and (4) screening and enrolment records. A descriptive analysis of the features was performed by the participating centre. Pearson's and Spearman's correlation coefficients were calculated to determine factors of recruitment success.

Results The highest recruitment rate was observed in Hospitals 3 and 6 (58.8 and 47.0 patients per month, respectively). All the study teams were multidisciplinary with a median of 15 members (range: 7-22). Only Hospitals 3, 5 and 6 had dedicated nursing staff appointed exclusively to this study. Moreover, in those three hospitals and in Hospital 9, the study coordinator performed exclusive functions as a research planner, and did not assume these functions for the other hospitals.

The univariate analysis showed a significant association between recruitment success and months of recruitment $(p=0.024)$, number of staff $(p<0.001)$, higher number of pharmacists $(p=0.005)$, infectious disease specialists $(p<0.001)$, the presence of microbiologist in the research team $(p=0.018)$ and specifically dedicated nursing staff $(p=0.036)$.

Conclusions The existence of broad multidisciplinary teams with staff dedicated exclusively to the study as well
Strengths and limitations of this study

- This study is a retrospective analysis of a prospective maintained database including all the potential candidates for a clinical trial.

- All participating hospitals in Spain enrolled in the REJUVENATE trial were included in this study.

- Other complex aspects in the development of a clinical trial were not analysed, such as patient followups or funding to protect research time.

- The lack of multivariable analysis to identify independent predictors of the recruitment rate was a limitation of the study.

as the implementation of a well-designed local patient assessment strategy were the essential optimisation factors for recruitment success in Spain.

Trial registration number NCT02655419; EudraCT 2015-002726-39; analysis of pre-screened patients.

\section{BACKGROUND}

Currently, evidence-based medicine relies on randomised clinical trials (RCTs), to show the highest level of evidence. However, these studies are tough, slow and expensive to perform; they are also often hindered by subject recruitment pitfalls. ${ }^{2}$ Sometimes, these trials cannot achieve the estimated population size. Furthermore, up to $86 \%$ of RCTs have failed to achieve recruitment targets within their specified time periods. ${ }^{34}$ This situation of suboptimal recruitment can result in underpowered and inconclusive studies, increased research costs, delays and unrepresentative sampling. 
Several strategies have been identified to try to solve these problems: establishing a psychological theory to identify strategies to increase research participation, ${ }^{5}$ defining determinants to predict and identify effective recruitment strategies or developing different surveys to identify strategies to overcome these problems. Isaksson et at constructed a questionnaire to identify factors that investigators and collaborators considered important. Kaur $e t a l^{7}$ also developed a survey to gather information on facilitators and barriers to recruitment that can be improved in other trials.

The extra time spent on recruitment in addition to normal duties was often mentioned as a major barrier to the investigator's involvement. ${ }^{8} 9$ Time-constraining factors include the administrative workloads associated with clinical trials, discussing the trial with the patients and patients' follow-ups, ${ }^{10}{ }^{11}$ in addition to regular training activities, and continuous study supervision. Thus, a multidisciplinary approach, having a trial coordinator, or funding to protect research time, ${ }^{12}$ are described as facilitators of successful recruitment.

As a result, common reasons for poor or slow recruitment in RCTs need to be considered at various levels, including the recruiting clinicians, trial centres, trial organisation and with the patients themselves ${ }^{5713}$ before starting the study.

In multicentre clinical trials, variations between centres or a combination of factors, such as centre size, centre initiation dates, investigator experience, site capabilities and infrastructure, institutional resources and target population access ${ }^{14}$ should be considered. The study design is also considered as one of the barriers that may prohibit a successful and 'on-time' recruitment. These include narrow eligibility criteria or complexity, which may lead to difficulties in explaining trials to patients. ${ }^{15}$

The European multicentre REJUVENATE trial successfully recruited the target sample size within the planned 18-month recruitment period. ${ }^{16}$ This prospective phase 2a open-label, single-arm study (NCT02655419; EudraCT 2015-002726-39) is the first interventional clinical trial conducted within the Innovative Medicines Initiative (IMI)-supported Combatting Bacterial Resistance in Europe-Carbapenem Resistance (COMBACTE-CARE) project. COMBACTE projects within the aztreonamavibactam (ATM-AVI) programme are characterised by their complexity in terms of patient identification. The first dose of study medication was administered within 24 hours after randomisation, and candidates came from multiple hospital clinical units in which different centres had organisational variations. The main objective of the REJVUENATE study was to determine the pharmacokinetics, safety and tolerability of ATM-AVI for the treatment of complicated intra-abdominal infections (cIAIs) in hospitalised adults. Between 19 May 2016 and 26 October 2017, patients were enrolled across 11 centres in France, Germany and Spain. Of the 40 patients enrolled in the REJUVENATE trial, $85 \%$ were recruited from Spanish centres while the remaining $15 \%$ were recruited from other countries participating in this trial. Nevertheless, not all the centres in Spain recruited the same number of patients, although all the hospitals included patients in the same inclusion period.

Understanding factors associated with recruitment success in Spain and differences between centres will facilitate the identification of key strengths or barriers. This will help in identifying measures to overcome these barriers, to facilitate the strengths in the future and how to improve recruitment in other centres. We performed this qualitative analysis with the aim of identifying the features of centres with the greatest potential to recruit patients and to detect potential factors associated with recruitment success. This could facilitate the performance of other clinical trials in Spain and other countries.

\section{MATERIAL AND METHODS \\ Study design and data sources}

This was a descriptive qualitative study. Trial features in each centre were retrospectively analysed from 19 May 2016 to 26 October 2017. Data were extracted from: (1) feasibility questionnaires completed by the centres in 2015 before patient enrolment to assess the centre's potential for patient enrolment; (2) delegation of responsibility records necessary to document all study staff members' important study-related duties; (3) prescreening records including an anonymised list of potentially eligible patients according to a subset of selection criteria (including demographic characteristics such as age and sex and medical characteristics) that could be verified retrospectively from available data in the patients' charts, and any reason (s) for pre-screening failure and (4) screening and enrolment records including pre-screened patients that signed the informed consent form but eventually met an exclusion criterion (screened patients), or when all eligibility criteria were met (enrolled patients). Principal investigators (PIs) and study coordinators were also asked to provide additional information if necessary. Data were extracted from the REJUVENATE study and approved by Hospital Universitario Virgen del Rocio Ethical committee with code ATM-AVI D4910C00009.

\section{Variables}

The collected factors that might affect enrolment were as follows: centre-related (centre size in terms of the number of beds as a proxy of complexity and capability, start date of recruitment and months of recruitment), and trial organisation-related (the PI's medical specialty, previous RCT experience, protected research time, competing RCTs, multidisciplinary approach, number of trained staff in each specialty, designation, dedication of a trial coordinator and dedicated nursing staff).

\section{Definitions}

Pre-screened patient: This was defined as a patient reviewed for eligibility. 


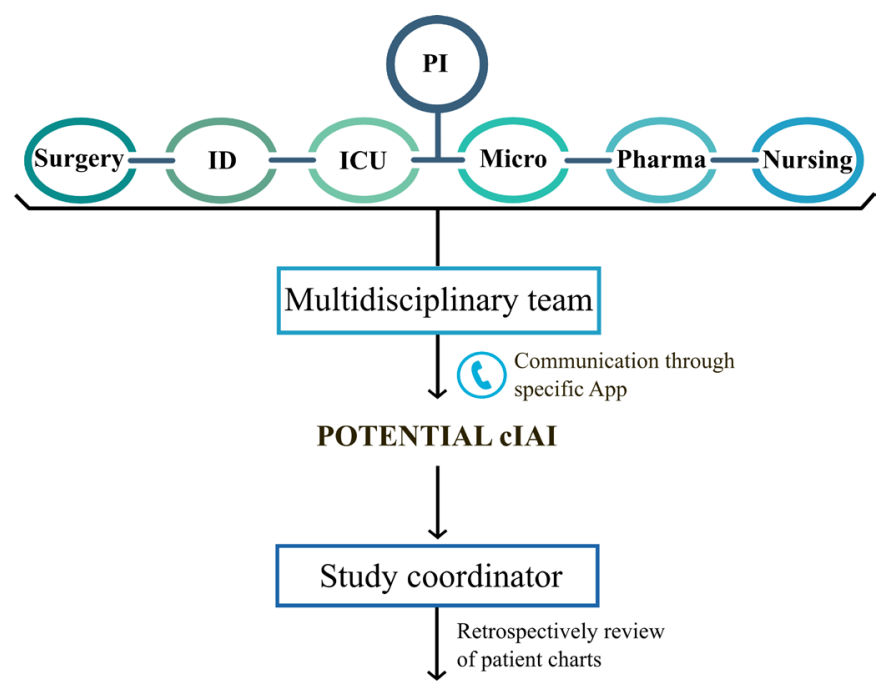

POTENTIAL CANDIDATE

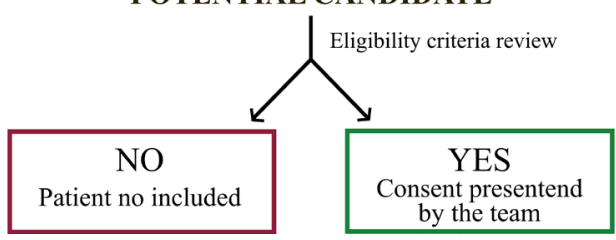

Figure 1 Flowchart of the recruitment process at Spanish sites. APP, application; cIAl, complicated intra-abdominal infection; ICU, intensive care unit; ID, infectious disease; Micro, microbiologists; Pharma, pharmacists; PI, principal investigator.

Screened patient: This was defined as patients that were reviewed for eligibility, who signed informed consent, but were not enrolled due to having met some exclusion criteria.

Enrolled patient: This was when a patient that was reviewed for eligibility, signed the informed consent, met all inclusion criteria and met no exclusion criteria.

Recruitment rate: This was defined as the number of patients enrolled at each centre per month.

Pre-screening rate: This was defined as the number of patients enrolled divided by the number of patients pre-screened.

Study coordinator: This was defined as a member of the study team who managed the RCT at the site level.

\section{Patient enrolment}

The scheme of the procedure for patient enrolment in Spain is summarised in figure 1 . Once a potential intraabdominal complication was identified through the screening of potential candidates, there was immediate communication to the research team and the study coordinator through an encoded app. At that point, the study coordinator checked from data available in the potential patient charts (pre-screened patients) to generate an anonymised list of potentially eligible patients, including reason(s) for pre-screening failure. Eventually, if a prescreened patient met all the inclusion criteria, and none of the exclusion criteria, the study would be presented to this potential candidate. If he/she agreed to participate and signed the informed consent (screened patient), the study coordinator would communicate it immediately to the multidisciplinary study team.

\section{Patient and public involvement}

Neither patients nor the public were directly involved in this research study.

\section{Participating centres}

All participating centres in Spain were included in this study, regardless of whether they enrolled patients or not. To adopt a similar approach within the national Spain territory, all study teams and sites received continuous training and support from the national coordinating team. The Spanish sites are part of the Spanish Network for Research in Infectious Diseases (REIPI, http://reipi.org/).

\section{Analysis and outcomes}

A descriptive analysis of the features was performed by each participating centre. We assessed the normality of distribution of variables using normal probability plots and the D'Agostino-Pearson normality test. The dependent variable for the analysis was the recruitment rate in each hospital. Pearson's or Spearman's rank correlation coefficients were used to measure the degree of association between two continuous variables, and the Student's t-test was used for categorical dichotomous variables. For all analyses, $p<0.05$ was considered statistically significant. Data analysis was carried out using R package stats in $\mathrm{R}^{17}$

\section{RESULTS}

\section{Centre (hospital) features}

Centre (hospital) features related to site logistics differed between participating hospitals and are shown in table 1 .

According to the centre size, participating hospitals ranged from having a large hospital capacity with $>1000$ beds (Hospitals 3,6 and 8 ), to lower capacity with $600-1000$ beds (Hospitals $1,5,7,9$ and 10), to those with $<600$ beds (Hospitals 2 and 4 ).

First, permission to proceed with enrolment was obtained for Hospitals 3, 5 and 6 in May 2016, with an enrolment period of 17 months, longer than the period for other hospitals. The recruitment period ended before Hospital 10 received the permission to proceed with recruitment. The screening of patients and pharmacy availability spanned $24 / 7$ in Hospitals 1, 4, 5 and 10. Competing RCTs in cIAI were in place in Hospitals 1, 2, 3, 4, 7 and 8.

\section{Patient enrolment}

A total of 837 patients were pre-screened from May 2016 to October 2017 in 8 out of the 10 Spanish participating centres. (table 2). Hospital 2 registered the highest number of pre-screened patients $(311 / 837$ patients, $37.7 \%)$, followed by Hospital $5(167 / 837,20.0 \%)$ and Hospital 6 (152/837, $18.4 \%$ ). Of the 31 enrolled patients, $32.2 \%$ and $25.8 \%$ came from Hospitals 3 and 6, respectively. No pre-screened patients were included for Hospitals 9 and 10. 
Table 1 Hospital features related to site logistics in the REJUVENATE study, Spain

\begin{tabular}{rrrllll} 
Hospital & No. of beds & $\begin{array}{l}\text { No. of months of } \\
\text { recruitment }\end{array}$ & Capacity 24/7 & Pharmacy 24/7 & $\begin{array}{l}\text { Belong to a } \\
\text { RN }\end{array}$ & $\begin{array}{l}\text { Competing RCTs } \\
\text { clAl }\end{array}$ \\
\hline 1 & 912 & 8 & Yes & Yes & No & 2 \\
\hline 2 & 470 & 15 & Yes & No & Yes & 1 \\
3 & 1233 & 17 & No & Yes & Yes & 1 \\
4 & 534 & 14 & Yes & Yes & No & 2 \\
5 & 960 & 17 & Yes & Yes & Yes & 0 \\
6 & 1350 & 17 & No & No & Yes & 0 \\
7 & 839 & 14 & Yes & No & Yes & 1 \\
8 & 1146 & 10 & No & No & Yes & 2 \\
\hline 9 & 728 & 11 & No & Yes & Yes & 0 \\
\hline 10 & 802 & 0 & Yes & Yes & No & 0 \\
\hline
\end{tabular}

cIAI, complicated intra-abdominal infection; RCTs, randomised clinical trials; REJUVENATE, Phase 2a open-label, multicentre study to investigate pharmacokinetics and safety and efficacy of the investigational monobactam/ $\beta$-lactamase inhibitor combination aztreonam/ avibactam in patients with complicated intra-abdominal infection; RN, research network.

The overall pre-screening rate was $3.7 \%$, ranging from $<1 \%$ (Hospitals 2 and 8) to $12.2 \%$ (Hospital 3) and excluded those in which the pre-screening rate was not applicable (NA). The recruitment rate ranged from zero patients per month (Hospitals 8, 9 and 10) to 58.8 patients per month (Hospital 3).

The highest recruitment rate was observed in Hospitals 3 and 6 (58.8 and 47.0 patients per month, respectively).

\section{Research teams}

The PI had prior experience in RCTs in all Hospitals. Only PIs from Hospitals 5 and 7 were surgeons, compared with infectious disease (ID) clinicians in other centres (table 3).

All the study teams were multidisciplinary and were made with a median of 15 (range: 7-22) persons (table 3). In general, at least one (range: 1-5 (Hospital 6)) surgeon was part of the research team, with no surgeon designated for
Hospitals 1 and 10. A median of four ID specialists were observed (range: $1-7$ ).

Only Hospitals 3, 5 and 6 had dedicated nursing staff appointed exclusively to this study.

In Hospitals 3, 5, 6 and 9 the study coordinator performed exclusive functions as a research planner and did not assume these functions at other hospitals (sub-investigator or study nurse).

\section{Factors associated with recruitment success}

The univariate analysis (table 4) showed a significant association between recruitment success and months of recruitment $(\mathrm{p}=0.024)$, number of staff $(\mathrm{p}<0.001)$, higher number of pharmacists $(p<0.001)$, ID specialists $(\mathrm{p}<0.001)$, specifically dedicated nursing staff $(\mathrm{p}=0.036)$ and the participation of clinical microbiologists $(\mathrm{p}=0.018)$. In a sub-analysis including only those hospitals

Table 2 Patient enrolment by participating hospitals in the REJUVENATE study, Spain

\begin{tabular}{|c|c|c|c|c|c|}
\hline \multirow[b]{2}{*}{ Hospital } & \multirow{2}{*}{$\begin{array}{l}\text { Pre-screened } \\
\mathrm{n}(\% \text { of total) }\end{array}$} & \multirow{2}{*}{$\begin{array}{l}\text { Screened } \\
\mathrm{n}(\% \text { of total) }\end{array}$} & \multirow[b]{2}{*}{ Enrolled } & \multirow[b]{2}{*}{ Pre-screening rate (\%) } & \multirow{2}{*}{$\begin{array}{l}\text { Recruitment rate (enrolled } \\
\text { patients per month) }\end{array}$} \\
\hline & & & & & \\
\hline 1 & $15(1.8)$ & $1(2.9)$ & $1(3.2)$ & 6.7 & 12.5 \\
\hline 3 & $82(9.9)$ & $11(32.4)$ & 10 (32.2) & 12.2 & 58.8 \\
\hline 4 & $46(5.6)$ & 4 (11.8) & $3(9.7)$ & 6.5 & 21.4 \\
\hline 6 & 152 (18.4) & 8 (23.6) & $8(25.8)$ & 4.8 & 47.0 \\
\hline 7 & $53(6.4)$ & $2(5.9)$ & $2(6.4)$ & 5.3 & 14.2 \\
\hline 8 & $11(1.3)$ & 0 & 0 & 0 & 0 \\
\hline 9 & 0 & 0 & 0 & NA & 0 \\
\hline
\end{tabular}

NA, not applicable; REJUVENATE, Phase 2a open-label, multicentre study to investigate pharmacokinetics and safety and efficacy of the investigational monobactam/ $\beta$-lactamase inhibitor combination aztreonam/avibactam in patients with complicated intra-abdominal infection. 
Table 3 Centre features related to the research team in the REJUVENATE study, Spain

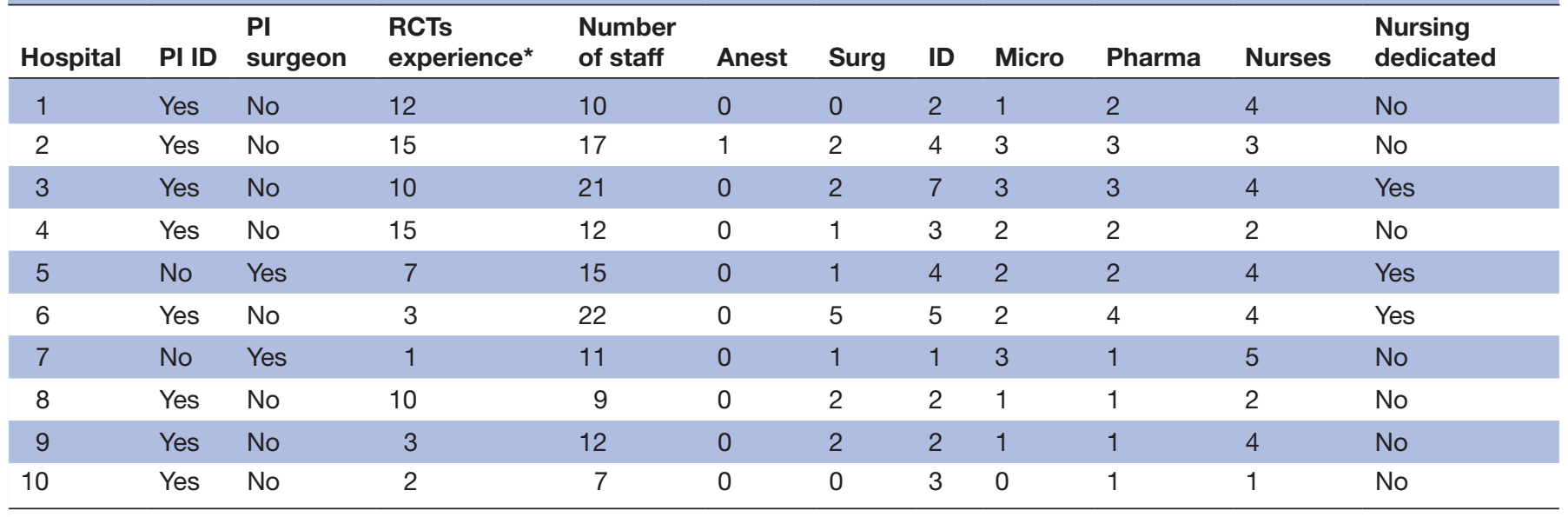

${ }^{*} \mathrm{RCTs}$ experience of the principal investigator in the last 5 years.

$\dagger$

$\ddagger$

Anest, anaesthetists; ID, infectious diseases; Micro, microbiologists; Pharma, pharmacists; PI, principal investigator; RCTs, randomised clinical trials; REJUVENATE, Phase $2 a$ open-label, multicentre study to investigate pharmacokinetics and safety and efficacy of the investigational monobactam/ $\beta$-lactamase inhibitor combination aztreonam/avibactam in patients with complicated intra-abdominal infection; Surg, surgeons.

Table 4 Univariate analysis of features associated with recruitment success in the REJUVENATE study, Spain

\begin{tabular}{|c|c|c|c|}
\hline Variable & $\mathbf{R}$ & $95 \% \mathrm{Cl}$ & P value \\
\hline Number of beds & 0.552 & -0.118 to 0.877 & 0.097 \\
\hline Months of recruitment & 0.700 & 0.125 to 0.923 & 0.024 \\
\hline RCT experience (PI last 5 years) & -0.143 & -0.709 to 0.534 & 0.691 \\
\hline Number of staff & 0.875 & 0.548 to 0.970 & 0.000 \\
\hline \multirow[t]{2}{*}{ Infectious disease } & 0.887 & 0.584 to 0.973 & 0.000 \\
\hline & rho & $95 \% \mathrm{Cl}^{\star}$ & \\
\hline Microbiologists & 0.719 & 0.105 to 0.947 & 0.018 \\
\hline Surgeons & 0.296 & -0.547 to 0.860 & 0.406 \\
\hline Nurses & 0.433 & -0.115 to 0.880 & 0.210 \\
\hline Pharmacy 24/7 & -0.131 & -33.59 to 30.05 & 0.898 \\
\hline Belongs to a research network & -0.851 & -63.34 to 40.99 & 0.477 \\
\hline PI-ID & 0.232 & -29.27 to 34.42 & 0.828 \\
\hline PI-Surgeon & -0.233 & -34.42 to 29.27 & 0.828 \\
\hline Nursing dedicated & -3.969 & -68.02 to -4.618 & 0.036 \\
\hline Study coordinator dedicated & -1.784 & -62.81 to 15.61 & 0.160 \\
\hline
\end{tabular}

${ }^{*} \mathrm{Cl}$ for Spearman's rho was calculated by bootstrapping (1000 replicates).

ID, infectious disease; PI, principal investigator; R, Pearson's correlation coefficient; RCT, randomised clinical trial; REJUVENATE, Phase $2 a$ open-label, multicentre study to investigate pharmacokinetics and safety and efficacy of the investigational monobactam/ $\beta$-lactamase inhibitor combination aztreonam/avibactam in patients with complicated intra-abdominal infection; rho, Spearman's correlation coefficient; $T$, Student's t-test. 
with permission to proceed with recruitment (Hospitals 1-9), the same features were significantly associated with recruitment success apart from the number of microbiologists $(\mathrm{p}=0.066)$ (online supplemental table $\mathrm{S} 1)$.

\section{DISCUSSION}

Recruitment of appropriate patients in a timely manner can be considered the Achilles' heel of a clinical successful trial. After analysing success in pre-screening and screening of patients in the Spain REJUVENATE study, ${ }^{16}$ this work revealed the importance of maintaining recruitment activity over time, the number of members in the research team and the importance of including pharmacists, ID doctors, clinical microbiologists and research nursing staff as members in a well-defined and engaged multidisciplinary team involved in the recruitment of patients.

Several different healthcare professionals working together positively impact the implementation of a clinical trial. Daykin $e t a l^{2}$ selected a purposive sample of active trials from the Health Technology Assessment Programme of the National Institute for Health Research (United Kingdom) portfolio between 2014 and 2015, to conduct semistructured interviews with several trial team members. They suggested that the role of trial staff and their underlying behaviours influence recruitment and retention practices. Thus, the incentives, the researchers' 'moral compass' and the staff's prior experience are key factors in improving recruitment. This approach to teamwork is of special importance in the context of anti-infective studies where severity of illness and urgency of treatment, among others, may add further complexity to the implementation of the study. In this context, acute patients may present to the hospital across several departments and at any time of the day and night. A close cooperation of a multidisciplinary team will be essential to dealing with the complexity of coordinating different aspects of these studies. These aspects include restrictive eligibility criteria, clinical status of patients, timing of surgery, adequate baseline microbiology specimens, complex dosing regimens and pharmacokinetics. Involving colleagues from different fields is considered important for enhancing the clinician researcher's motivation. In addition, maintaining recruitment activity over time has been shown to maximise recruitment because it maintains the enthusiasm for recruiting the subjects. ${ }^{48}$ In our study, the number of patients recruited was higher in those sites with a higher number of investigators: Hospitals 3 and 6 had 21 and 22 investigators, respectively.

Other published works focusing on the challenges of recruitment during prospective trials ${ }^{18-22}$ discuss the possibility of monetary incentives for patients, and the differences in views, beliefs and experiences of team members are also considered. Donovan $e t a l^{21}$ addressed the problems from research nurses' point of view. The extra workload associated with participation in clinical trials is also a limiting factor for patient recruitment. ${ }^{323}$
Thus, the meta-analysis performed by Treweek $e t a l^{3}$ shows the importance of the exclusive dedication to research of participating clinicians, as well as the involvement of research networks. The use of staff with protected time for research has been previously shown to improve recruitment performance in RCTs. ${ }^{412}$ In the REJUVENATE study, top-recruiting sites in Spain (Hospitals 3, 5 and 6) included nurses specifically dedicated to the project. The two sites (Hospitals 3 and 6) with the highest recruitment rate were those that had the least largest number of staff including nursing staff exclusively dedicated to the study

In the current work, teams comprising of more investigators across more departments had a higher recruitment rate than others. Furthermore, teams composed of more pharmacists, ID specialists and microbiologists showed a higher recruitment success. In addition, toprecruiting sites included nurses specifically dedicated to the project. In a well-endowed and coordinated multidisciplinary team, the distribution of tasks is clear and more acceptable by each team member. However, in teams with limited staff or without good coordination, the inclusion of the first patient is so difficult that it does not motivate the inclusion of many more patients. ${ }^{48} \mathrm{Lack}$ of active involvement of the PI is another possible reason why no patient was included in several Spanish hospitals, reflecting the importance of the clinician's engagement as a crucial step in the recruitment process. ${ }^{8}$

Pre-screening activities to determine the initial patients' eligibility into a clinical study is common practice and is considered a great strategy to optimise any patient recruitment campaign. ${ }^{24}$ These activities are often performed variably across multicentre global studies and require a high level of staff resources to deliver. Pre-screening may involve several initiatives across a hospital site to ensure that no potential patient is missed, such as daily database surveillance or theatre/admission lists. In addition, it will allow the researcher to assess the recruitment plan and to detect early unanticipated barriers to recruitment, such as an individual's ability to come to the research site multiple times.

However, recruiting appropriate participants for a specific study under a tight timeline, especially in acute settings, requires strategic planning to increase efficiency, reduces the enrolment period and minimises protocol deviations. Therefore, with an effective pre-screening plan that should include a clear definition, we can ensure that pre-screened patients meet the more general inclusion criteria (eg, adult hospitalised to be operated for cIAI). Thus, sites can make this process more efficient and ensure that they identify the right candidates for their study. During the pre-screening period, most of those subjects who may not meet a simple inclusion criterion for the study can be excluded.

The implementation of pre-screening procedures as part of the subject selection and recruitment process in the REJUVENATE study ${ }^{16}$ was highly promoted by the national coordinating team. Although there were differences across the participating sites, defined strategies 
were based on very close coordination between surgery and ID departments for the methodical assessment of the list of surgery patients. The pre-screening process was efficiently achieved in sites that favoured the active involvement and daily communication between well-endowed, trained team of surgeons and trained ID clinical team. The use of instant messaging technology facilitated the early detection of candidates for the study. Following the described pre-screening approach, since phase 3 (REVISIT study (NCT03329092; EudraCT 2017-00274268) of the ATM-AVI programme that started in April 2018), Spanish sites have randomised seven patients so far (28\% of the total).

The main limitations of this study are related to its retrospective design, although all the information was obtained from a prospective maintained database. Second, all cases were obtained from the same country, Spain, which could lead to potential bias due to different healthcare systems in which there may be different recruitment problems or interests from patients and healthcare professionals. Finally, other complex aspects in the development of a trial, such as patients' follow-ups or funding to protect research time were not analysed in addition to the lack of multivariable analysis to identify independent predictors of recruitment rate.

The strengths of the study include the large representation in a whole country. The study had a multicentre setting in Spain, which may have potentially limited the generalisability of the findings in other countries. This also provided assurance that the recruitment problems included represented a high variety of the factors associated with the inclusion of patients in a trial.

To the best of our knowledge, this study could be one of the first exploring recruitment achieved in relation to the patient evaluation process and trial features at participating centres from the first interventional clinical trial ${ }^{16}$ conducted within the IMI-supported COMBACTE-CARE project. However, we did not analyse other complex aspects in the development of the trial, such as retention in the study and attitudinal issues.

\section{CONCLUSION}

In conclusion, the creation of a well-endowed multidisciplinary team with staff dedicated exclusively to the study as well as the implementation of a well-designed local patient assessment strategy are cornerstones of the success of patient recruitment in antibiotic clinical trials.

\footnotetext{
Author affiliations

${ }^{1}$ Department of Surgery, Hospital Universitario Virgen del Rocio, Sevilla, Spain ${ }^{2}$ Department of Microbiology, Hospital Universitario Virgen del Rocio, Sevilla, Spain ${ }^{3}$ Clinical Research and Clinical Trials Unit, Hospital Universitario Virgen del Rocio, Sevilla, Spain

${ }^{4}$ Department of Infectious Diseases, Hospital Universitario Virgen del Rocío, Sevilla, Spain

${ }^{5}$ Global Antibiotic R\&D Partnership (GARDP), Geneva, Switzerland

${ }^{6}$ Department of Infectious Diseases, Reina Sofia University Hospital, Cordoba, Spain

${ }^{7}$ Department of Surgery, Hospital Universitario Virgen Macarena, Sevilla, Spain

${ }^{8}$ Department of Infectious Diseases, Hospital del Mar, Barcelona, Spain
}

${ }^{9}$ Department of Surgery, Hospital Universitari Son Espases, Palma de Mallorca, Spain

${ }^{10}$ Department of Infectious Diseases, MutuaTerrassa Group, Terrassa, Spain

${ }^{11}$ Department of Infectious Diseases, Hospital Universitario Cruces, Barakaldo, Spain

${ }^{12}$ IMIBIC/Reina Sofia Hospital, Cordoba, Spain

Twitter Luis Tallón-Aguilar @|tallona

Acknowledgements IMI G.A. 115620

Contributors RMJ-R, GM-G and SJ-J were responsible for the design of the study, data collection and data analysis. RMJ-R, GM-G, SJ-J, CMR-F, LT-A, CR-0, JP, AL, AC, JL-R, SG-Z, JB-P, LB-P, JMM, JT-C and JMC reviewed the drafts and final versions of the manuscript. RMJ-R is responsible for the overall content, accepts full responsibility for the work, has access to the data and controlls the decision to publish.

Funding The REJUVENATE trial was supported by Innovative Medicines Initiative (IMI) Combatting Bacterial Resistance in Europe-Carbapenem-Resistance COMBACTE-CARE WP2B (G.A. 115620). Plan Nacional de I+D+i 2013-2016 (Instituto de Salud Carlos III, Subdirección General de Redes y Centros de Investigación Cooperativa, Ministerio de Ciencia, Innovación y Universidades, Spanish Network for Research in Infectious Diseases-REIPI) with the grants RD16/0016/009; RD16/0016/0008; RD16/0016/0001; RD16/0016/0015; RD16/0016/0004; RD16/0016/0012. The study was supported by the SCReNSpanish Clinical Research Network (PT13/0002/0010-PT17/0017/0012) from the National $R+D+$ I Plan of the Institute of Health Carlos III (Ministerio de Ciencia, Innovación y Universidades).

Competing interests Alison Luckey was Pfizer UK Ltd employee at the time of the study.

Patient consent for publication Not applicable.

Ethics approval This study involves human participants. Data were extracted from the REJUVENATE study and approved by Hospital Universitario Virgen del Rocio Ethical committee with code ATM-AVI D4910C00009. Participants gave informed consent to participate in the study before taking part.

Provenance and peer review Not commissioned; externally peer reviewed.

Data availability statement Data are available upon reasonable request. Access to data: upon request to the corresponding author. Deidentified centre's data will be made available to researchers whose proposals meet the research criteria. To gain access, data requestors must comply with a data access agreement.

Supplemental material This content has been supplied by the author(s). It has not been vetted by BMJ Publishing Group Limited (BMJ) and may not have been peer-reviewed. Any opinions or recommendations discussed are solely those of the author(s) and are not endorsed by BMJ. BMJ disclaims all liability and responsibility arising from any reliance placed on the content. Where the content includes any translated material, BMJ does not warrant the accuracy and reliability of the translations (including but not limited to local regulations, clinical guidelines, terminology, drug names and drug dosages), and is not responsible for any error and/or omissions arising from translation and adaptation or otherwise.

Open access This is an open access article distributed in accordance with the Creative Commons Attribution Non Commercial (CC BY-NC 4.0) license, which permits others to distribute, remix, adapt, build upon this work non-commercially, and license their derivative works on different terms, provided the original work is properly cited, appropriate credit is given, any changes made indicated, and the use is non-commercial. See: http://creativecommons.org/licenses/by-nc/4.0/.

\section{ORCID iDs}

Rosa M Jimenez-Rodriguez http://orcid.org/0000-0001-8587-8768 Julian Torre-Cisneros http://orcid.org/0000-0003-1529-6302

\section{REFERENCES}

1 Caldwell PHY, Hamilton S, Tan A, et al. Strategies for increasing recruitment to randomised controlled trials: systematic review. PLOS Med 2010;7:e1000368.

2 Daykin A, Clement C, Gamble C, et al. 'Recruitment, recruitment, recruitment' - the need for more focus on retention: a qualitative study of five trials. Trials 2018;19:76.

3 Treweek S, Lockhart P, Pitkethly M, et al. Methods to improve recruitment to randomised controlled trials: cochrane systematic review and meta-analysis. BMJ Open 2013;3:e002360. 
4 McDonald AM, Knight RC, Campbell MK, et al. What influences recruitment to randomised controlled trials? A review of trials funded by two UK funding agencies. Trials 2006;7:9.

5 Sheridan R, Martin-Kerry J, Hudson J, et al. Why do patients take part in research? an overview of systematic reviews of psychosocial barriers and facilitators. Trials 2020;21:259.

6 Isaksson E, Wester P, Laska AC, et al. Identifying important barriers to recruitment of patients in randomised clinical studies using a questionnaire for study personnel. Trials 2019;20:618.

7 Kaur G, Smyth RL, Williamson P. Developing a survey of barriers and facilitators to recruitment in randomized controlled trials. Trials 2012;13:218

8 Fletcher B, Gheorghe A, Moore D, et al. Improving the recruitment activity of clinicians in randomised controlled trials: a systematic review. BMJ Open 2012;2:e000496.

9 Sin J, Henderson C, Spain D, et al. What factors influence successful recruitment of siblings of individuals with first episode psychosis to e-health interventions? A qualitative study. Health Expect 2017;20:696-704.

10 Mahmud A, Zalay O, Springer A, et al. Barriers to participation in clinical trials: a physician survey. Curr Oncol 2018;25:119-25.

11 Tournoux C, Katsahian S, Chevret S, et al. Factors influencing inclusion of patients with malignancies in clinical trials. Cancer 2006;106:258-70.

12 Kenyon S, Rhodes A, Taylor D. A recipe for successful recruitment to a randomised controlled trial. MDIRS Midwifery Digest 2005;15:16e19.

13 Huang GD, Bull J, Johnston McKee K, et al. Clinical trials recruitment planning: a proposed framework from the clinical trials transformation initiative. Contemp Clin Trials 2018;66:74-9.

14 Gkioni E, Rius R, Dodd S, et al. A systematic review describes models for recruitment prediction at the design stage of a clinical trial. J Clin Epidemiol 2019;115:141-9.
15 Skea ZC, Treweek S, Gillies K. 'It's trying to manage the work': a qualitative evaluation of recruitment processes within a UK multicentre trial. BMJ Open 2017;7:e016475.

16 Cornely OA, Cisneros JM, Torre-Cisneros J, et al. Pharmacokinetics and safety of aztreonam/avibactam for the treatment of complicated intra-abdominal infections in hospitalized adults: results from the REJUVENATE study. J Antimicrob Chemother 2020;75:618-27.

17 Team RC. R: a language and environment for statistical computing, 2013. Available: https://www.r-project.org [Accessed 17 May 2020]

18 Campillo-Gimenez B, Buscail C, Zekri O, et al. Improving the prescreening of eligible patients in order to increase enrollment in cancer clinical trials. Trials 2015;16:15.

19 Adams M, Caffrey L, McKevitt C. Barriers and opportunities for enhancing patient recruitment and retention in clinical research: findings from an interview study in an NHS academic health science centre. Health Res Policy Sys 2015;13:8.

20 de Salis I, Tomlin Z, Toerien M, et al. Using qualitative research methods to improve recruitment to randomized controlled trials: the quartet study. J Health Serv Res Policy 2008;13:92-6.

21 Donovan JL, Paramasivan S, de Salis I, et al. Clear obstacles and hidden challenges: understanding recruiter perspectives in six pragmatic randomised controlled trials. Trials 2014;15:5.

22 Lawton J, Snowdon C, Morrow S, et al. Recruiting and consenting into a peripartum trial in an emergency setting: a qualitative study of the experiences and views of women and healthcare professionals. Trials 2016;17:195.

23 Prout $\mathrm{H}$, Butler $\mathrm{C}$, Kinnersley $\mathrm{P}$, et al. A qualitative evaluation of implementing a randomized controlled trial in general practice. Fam Pract 2003;20:675-81.

24 Good MJ, Lubejko B, Humphries K, et al. Measuring clinical trialassociated workload in a community clinical oncology program. $J$ Oncol Pract 2013;9:211-5 